\title{
Efecto de los residuos fibrosos de avena (Avena sativa) y caraotas (Phaseolus vulgaris) sobre la actividad in vitro de las disacaridasas intestinales en ratas
}

\author{
I \\ 1 \\ 1 \\ PERSPECTIVAS EN NUTRICIÓN HUMANA \\ ISSN 0124-4108 \\ Escuela de Nutrición y Dietética, Universidad de Antioquia. Medellín, Colombia
}

Vol. 20, N. ${ }^{\circ}$ 2, julio-diciembre de 2018, p. 131-143.

DOI:10.17533/udea.penh.v20n2a02

Artículo recibido: 26 de abril de 2018

Aprobado: 18 de octubre de 2018

Ana Virginia Ávila*; Mirla Morón²; Andrés Carmona³; Pablo Hernández4; Ramón Infante; Omar García ${ }^{6}$; Francisco Torrealba ${ }^{7}$

$1^{*}$ Autor de correspondencia. Magíster en Nutrición. Licenciada en Nutrición y Dietética. Profesora asociada de la Cátedra de Nutrición Humana, Escuela de Nutrición y Dietética, Facultad de Medicina, Universidad Central de Venezuela. Caracas-Venezuela. avila.anav@gmail.com

2 Magíster en Nutrición. Licenciada en Nutrición y Dietética. Profesora asociada de la Cátedra de Nutrición Humana, Escuela de Nutrición y Dietética, Facultad de Medicina, Universidad Central de Venezuela. Caracas-Venezuela. mormir1811@ hotmail.com

3 Doctor en Biología Celular. Profesor titular, Instituto de Biología Experimental, Facultad de Ciencias, Universidad Central de Venezuela. Caracas-Venezuela. ajcarmona @gmail.com

4 Magíster en Nutrición. Licenciado en Nutrición y Dietética. Profesor Instructor de la Cátedra de Nutrición Humana, Escuela de Nutrición y Dietética, Facultad de Medicina, Universidad Central de Venezuela. Caracas-Venezuela. doctuscumliber@gmail.com

5 Doctor en Bioquímica. Profesor titular de la Cátedra de Bioquímica, Escuela de Nutrición y Dietética, Facultad de Medicina, Universidad Central de Venezuela. Caracas-Venezuela. infanterster@gmail.com

6 Magíster en Ciencia y Tecnología de los Alimentos. Licenciado en Nutrición y Dietética. Profesor asociado de la Cátedra de Tecnología de Alimentos, Escuela de Nutrición y Dietética, Facultad de Medicina, Universidad Central de Venezuela. Caracas-Venezuela. omar.garcia1908@gmail.com

7 Médico cirujano. Escuela de Medicina Luis Razetti, Facultad de Medicina, Universidad Central de Venezuela. Caracas-Venezuela. torrfran.javier@gmail.com

Cómo citar este artículo: Ávila AV, Morón M, Carmona A, Hernández P, Infante R, García O, et al. Efecto de los residuos fibrosos de avena (Avena sativa) y caraotas (Phaseolus vulgaris) sobre la actividad in vitro de las disacaridasas intestinales en ratas. Perspect Nutr Humana. 2018;20:131-43. DOI: 10.17533/udea.penh.v20n2a02 
Resultados: la mayor actividad enzimática se registró en la región intestinal media para cada enzima $(p<0,05)$. El orden de actividad enzimática en $\mathrm{mg}$ glucosa/mg proteína/min fue maltasa $(0,149)$ sacarasa $(0,096)$ y lactasa $(0,014)$ $(p<0,05)$. La maltasa fue inhibida en mayor medida por el residuo de caraota; la sacarasa, por el residuo de avena; $y$ la lactasa, por ambos. Conclusiones: la adición de fibra purificada de avena y caraota produjo una disminución significativa de la actividad in vitro de las disacaridasas intestinales, especialmente en presencia del residuo de caraota.

Palabras clave: fibra dietética, disacaridasas, sacarasa, maltasa, lactasa.

\section{Effect of the Fibrous Residues of Avena (Avena sativa) and Black Beans (Phaseolus vulgaris) on the in vitro Activity of Intestinal Disacaridases in Rats}

\section{Abstract}

Background: Intestinal disaccharidases can be partially inhibited or stimulated in the presence of fiber. Objective: To evaluate the effect of fibrous residues of oats (Avena sativa) and black beans (Phaseolus vulgaris) on the "in vitro" activity of the intestinal disaccharidases. Materials and Methods: 15 Sprague Dawley rats, were divided into three groups: control, fed with bean flour, and fed with oatmeal flour for 21 days. Homogenate was obtained by scraping the mucosa. The determination of enzymatic activity of the disaccharidases was measured by the enzymatic method, in the presence of its natural substrate and with addition of the fibrous residues obtained from the oatmeal and black beans, in concentration of $2.5 \%$ (W/V). Results: The highest enzymatic activity was recorded in the middle intestinal region for each enzyme $(p<0.05)$. The order of enzymatic activity in $\mathrm{mg}$ glucose / $\mathrm{mg}$ protein $/ \mathrm{min}$ was maltase $(0.149)$ sucrase $(0.096)$ and lactase $(0.014)(p<0.05)$. Maltase was inhibited to a greater extent by bean residue; sucrase by oat residue and lactase by both. Conclusion: The addition of purified fiber of oats and bean produced a significant decrease in the in vitro activity of the intestinal disaccharidases, especially in the presence of the bean residue..

Keywords: Dietary fiber, disaccharidases, sucrase, maltase, lactase.

\section{INTRODUCCIÓN}

Entre 1966 y 1972, Denis Burkitt se basó en el trabajo de Peter Cleave, GD Campbell, Hugh Trowell, Neil Painter y Alec Walker para proponer que las dietas bajas en fibra aumentan el riesgo de enfermedad cardiovascular, obesidad, diabetes, caries dental, diversos trastornos vasculares y afecciones del intestino grueso, como cáncer, apendicitis y diverticulosis. El simple hecho de agrupar estas enfermedades como una causa común fue innovador. Proponer la fibra como la clave estimuló muchas investigaciones, pero también causó controversia (1).
Las definiciones más recientes de fibra dietética resaltan el nivel de polimerización de los compuestos catalogados como fibra, incluyendo desde los monosacáridos hasta los polisacáridos no amiláceos. El Codex Alimentarius la define como sustancias que presentan una función fisiológica de fibra (2), mientras que el Instituto de Medicina de Estados Unidos reserva el término de fibra alimentaria solo para aquellas fibras que son intrínsecas e intactas (3).

Las dietas con un elevado contenido en fibra requieren más tiempo de masticación. A nivel del estómago y como consecuencia de su viscosidad, enlentecen el vaciamiento gástrico y aumentan su 
distensión, prolongando la sensación de saciedad. En el intestino delgado incrementa el tiempo de tránsito y aumenta el espesor de la capa de agua, lo que provoca una disminución en la absorción de carbohidratos, lípidos y proteínas (4).

En relación con la digestión de los carbohidratos en el borde en cepillo de los enterocitos que contiene enzimas hidrolíticas, maltasa, $\alpha$-dextrinasa, lactasa y sacarasa, que descomponen los oligosacáridos hasta convertirlos en sus monosacáridos constituyentes, las enzimas pueden ser inhibidas o estimuladas parcialmente en presencia de fibra (5). Normalmente, los disacáridos son degradados a monosacáridos por disacaridasas (p. ej., lactasa, maltasa, isomaltasa, sacarasa [invertasa]) las cuales se encuentran en el borde de cepillo de las células absorbentes en el intestino delgado. La malabsorción de estos disacáridos es una de las causas frecuentes de diarrea osmótica. La fermentación bacteriana de los hidratos de carbono en el colon produce gases (hidrógeno, dióxido de carbono y metano), que causan flatulencia excesiva, distensión y dolor abdominal. Esta intolerancia a los carbohidratos se ha definido como síndrome de malabsorción (4).

La inhibición en la actividad de las enzimas pancreáticas e intestinales ha sido reportada en varios estudios in vitro $(4,6)$. Estudios recientes, que utilizaron modelos experimentales, reportaron la similitud entre la actividad hidrolítica de las disacaridasas intestinales entre humanos y ratas, proponiendo este modelo como una manera práctica para evaluar el efecto de polisacáridos indigeribles sobre la actividad de las enzimas digestivas (7).

En la presente investigación se escogieron dos alimentos que forman parte de la dieta del venezolano: la caraota como fuente de fibra insoluble y la avena en hojuelas como fuente de fibra soluble. El objetivo del presente trabajo fue evaluar el efecto de los residuos fibrosos de avena (Avena sativa) y caraotas (Phaseolus vulgaris) sobre la actividad in vitro de las disacaridasas intestinales en ratas.

\section{MATERIALES Y MÉTODOS}

\section{Ensayo biológico}

Dietas. En la elaboración de las dietas experimentales se utilizó el grano de Phaseolus vulgaris (conocido en Venezuela como caraota), variedad tacarigua, procedente de un mercado local. Se limpiaron los granos de impurezas y se añadieron en agua, en una proporción de 3:1. Se procedió a la cocción en una olla de presión durante una hora; luego se secaron en una estufa con circulación de aire, a una temperatura de $60{ }^{\circ} \mathrm{C}$ durante 24 horas para su posterior molienda. La avena en hojuelas se obtuvo de un mercado local. Se molió cruda y se tamizó para ser añadida con los otros ingredientes de la dieta experimental.

Determinación de fibra dietética total. La determinación del contenido de fibra dietética total en las muestras de harina de avena y de harina de caraotas negras se realizó utilizando el método enzimático de Prosky et al. (8). Se hizo un pool con el residuo fibroso obtenido por este método, con la finalidad de obtener la cantidad suficiente por cada harina, para la realización de los experimentos.

Animales. Se emplearon 15 ratas macho de la cepa Sprague Dawley, de 25 días de nacidas, con un peso inicial promedio de $85,8 \pm 14,1 \mathrm{~g}$. Se dividieron al azar en tres grupos de cinco ratas cada uno: un grupo control, alimentado con una dieta basal semipurificada, y dos grupos experimentales, que se diferenciaron del control al sustituir un $15 \%$ del porcentaje de almidón añadido a la dieta por una fuente de fibra insoluble (harina de caraota) y fibra soluble (harina de avena) (Tabla 1). Los animales 
se mantuvieron en jaulas individuales, con comida y agua ad libitum y bajo condiciones controladas de luz y temperatura, por un período de 21 días.

Procedimiento experimental. Después de 21 días de experimentación, los animales fueron sacrificados bajo una atmósfera saturada de éter etílico. Se procedió a la remoción del intestino delgado, irrigándolo periódicamente con solución fisiológica fría de 0,15 M de cloruro de sodio.

Se lavaron los intestinos usando esta misma solución con el fin de eliminar los restos de alimentos. Seguidamente, se dividieron en tres porciones iguales (proximal, media y distal). Cada porción de intestino fue abierta exponiendo la región luminal y se procedió inmediatamente al raspado de la mucosa. El material intestinal obtenido fue suspendido en un volumen equivalente a cuatro veces su peso en una solución fría de cloruro de sodio, que contenía $5 \mathrm{mM}$ de fluoruro de fenilmetilsulfonilo (PMSF) y $25 \mu \mathrm{g} / \mathrm{mL}$ de leupeptina. Estos últimos son inhibidores de proteasas. Luego se homogenizó en un Potter-Elvehjem (luz: 0,006 pulgadas) durante dos minutos, con 10 pases a una velocidad aproximada de 1725 rpm, luego los homogeneizados se centrifugaron a 5000 rpm por 10 minutos a $4 \stackrel{\circ}{\mathrm{C}}$. Las fracciones de sobrenadantes obtenidas fueron transferidas a viales y congeladas inmediatamente a $-70 \stackrel{\circ}{ } \mathrm{C}$ hasta su posterior análisis.

Para la determinación de la concentración de proteínas, se utilizó el método de Lowry et al. (9). En la determinación de la actividad enzimática de las disacaridasas (maltasa, sacarasa y lacta$\mathrm{sa}$ ), en presencia de su sustrato natural y con la adición de los residuos fibrosos de avena y caraota, se incubaron los homogeneizados con el sustrato correspondiente a la enzima, a una concentración final de $38 \mathrm{mM}$. Luego se añadieron los residuos de fibra dietética total obtenida en las muestras de harina de caraotas (Phaseolus vulgaris), y en la harina de avena (Avena sativa), para obtener una concentración final de $2,5 \%$ de residuos fibrosos $(\mathrm{P} / \mathrm{V})$.

La determinación de la actividad de la maltasa y la sacarasa se efectuó en presencia de buffer citrato de sodio $0,1 \mathrm{M}, \mathrm{pH} 6,3$, mientras que para determinar la actividad de la lactasa se ajustó el pH del buffer a 5,5. El tiempo de incubación de la reacción fue de 5 minutos a una temperatura de $37 \stackrel{\circ}{\mathrm{C}}$. Posteriormente, la reacción se detuvo al colocar los tubos en un baño a $90 \stackrel{\circ}{\mathrm{C}}$ por 5 minutos. Finalmente, para la determinación de la actividad de las disacaridasas estudiadas se utilizó el método enzimático glucosa peroxidasa (10).

Tabla 1. Composición de las dietas experimentales

\begin{tabular}{|c|c|c|c|}
\hline \multirow[b]{3}{*}{ Ingredientes } & \multirow{2}{*}{\multicolumn{3}{|c|}{ Tipo de dieta }} \\
\hline & & & \\
\hline & Control & Harina avena & Harina caraota \\
\hline Caseína (g/100 g de mezcla)a & 18 & 18 & 18 \\
\hline Aceite de maíz ( $\mathrm{g} / 100 \mathrm{~g}$ de mezcla) & 8 & 8 & 8 \\
\hline Mezcla de vitaminas (g/100 g de mezcla) ${ }^{b}$ & 1 & 1 & 1 \\
\hline Mezcla de minerales ( $\mathrm{g} / 100 \mathrm{~g}$ de mezcla $)^{\mathrm{b}}$ & 4 & 4 & 4 \\
\hline Almidón de maíz (g/100 g de mezcla) & 69 & 54 & 54 \\
\hline Harina de avena ( $\mathrm{g} / 100 \mathrm{~g}$ de mezcla) & - & 15 & - \\
\hline Harina de caraota negra ( $\mathrm{g} / 100 \mathrm{~g}$ de mezcla) & - & - & 15 \\
\hline
\end{tabular}

a. Caseína libre de vitaminas de Harlan-Teklad. Madison Wisconsin USA.

b. Mezcla AIN-76 de Harlan-Teklad. 


\section{Análisis estadístico}

Los resultados obtenidos fueron procesados estadísticamente mediante análisis de varianza de una vía y factorial, seguido de la prueba de Tukey, con un nivel de significancia de 0,05, además de las determinaciones de media, desviación estándar y error estándar, utilizando el programa estadístico SPSS ${ }^{\circledR}$ en su versión 21.

\section{Consideraciones éticas}

Se siguieron las normas bioéticas internacionales para el manejo de los animales de experimentación (11). La presente investigación fue avalada por el Comité Independiente de Bioética para la Investigación del Centro Nacional de Bioética bajo el número CIBI-CENABI-09/2018.

\section{RESULTADOS}

\section{Determinación de fibra dietética}

En la tabla 2 se muestran los valores de fibra dietética total en las muestras de avena en hojuelas y de caraotas negras cocidas obtenidas por el método enzimático descrito por Prosky et al. (8); los valores se expresaron en porcentaje por cada $100 \mathrm{~g}$ de alimento.

El valor de fibra dietética total que se obtuvo para la avena en hojuelas fue de 9,9\% y para la caraota negra en granos cocidos fue de $9,0 \%$, usando la variedad tacarigua.

Tabla 2. Contenido de fibra dietética total en las muestras de harina de avena y harina de caraotas negras

\begin{tabular}{lc}
\hline & Fibra dietética total $(\mathrm{g} / 100)$ \\
\hline \multirow{2}{*}{ Muestras analizadas } & $\mathrm{n}=10$ \\
\cline { 2 - 2 } & $\mathrm{X} \pm \mathrm{DE}$ \\
\hline Avena en hojuelas (g/100) & $9,9 \pm 1,5$ \\
Caraotas negras (cocidas) $(\mathrm{g} / 100)$ & $9,0 \pm 1,4$ \\
\hline
\end{tabular}

$\mathrm{X} \pm \mathrm{DE}$ promedio más menos desviación estándar.

\section{Actividad in vitro de la maltasa}

Los valores de la actividad específica de la maltasa en los tres segmentos intestinales estudiados, según el tipo de dieta suministrada y por tipo de tratamiento aplicado, se observan en la tabla 3. En la sección proximal y media se observó que la adición de los residuos fibrosos de avena y caraota al medio de incubación produjeron un descenso significativo $(p<0,05)$ en la actividad enzimática de la maltasa para todas la dietas previas. Sin embargo, la disminución promedio fue mayor para el agregado de caraota que para la avena en las secciones proximal $(67,6 \%$ vs. $33,7 \%)$ y media $(62,1 \%$ vs. $45,7 \%$ ), respectivamente. La mayor disminución se observó en los animales alimentados previamente con fibra de caraota cuando se añadió residuo fibroso de caraota a la sección del intestino proximal $(70,1 \%)$ y a la sección media $(67,3 \%)$.

En la sección distal, la adición de residuo fibroso de avena y caraota produjo un descenso aproximadamente del $58 \%$ en la actividad de la maltasa de las ratas alimentadas previamente con avena, mientras que la adición de residuos de fibra de caraota ocasionó una disminución $(51,7 \%)$ en la actividad de la maltasa en ratas alimentadas sin fibra.

\section{Actividad in vitro de la sacarasa}

La actividad enzimática de la sacarasa en presencia de su sustrato natural, y cuando a esta reacción se añadió el residuo fibroso de caraota y de avena, en las tres secciones intestinales estudiadas, se muestra en la tabla 4.

En la sección proximal se observó que la adición de residuo fibroso de caraota al medio de reacción produjo una mayor disminución de la actividad enzimática en los tres tipos de dieta, en especial en la dieta previa con caraota $(51,8 \%)$. La adición de avena, por su parte, produjo una disminución similar al añadido de caraota en las dietas sin fibra $(43,8 \%)$ y con fibra de avena $(50,0 \%)$. 
Efecto de residuos fibrosos sobre la actividad in vitro de las disacaridasas

Tabla 3. Actividad enzimática in vitro de la maltasa en las tres secciones intestinales, por diferentes fuentes enzimáticas, según tipo de tratamiento

\begin{tabular}{|c|c|c|c|c|c|}
\hline & & \multirow{4}{*}{ Grupos (fuente enzimática) } & \multicolumn{3}{|c|}{$\begin{array}{l}\text { Tratamiento } \\
\text { Actividad enzimática en } \mathrm{mg} \text { de glucosa/mg proteína/min }\end{array}$} \\
\hline & & & Control & Avena & Caraota \\
\hline & & & $X \pm D E$ & $X \pm D E$ & $\mathrm{X} \pm \mathrm{DE}$ \\
\hline & & & $n=3$ & $n=3$ & $n=3$ \\
\hline \multirow{9}{*}{$\begin{array}{l}\text { Sección } \\
\text { Intestinal }\end{array}$} & \multirow{3}{*}{ Proximal } & Sin fibra & $0,191 \pm 0,009^{a}$ & $0,140 \pm 0,003^{b}$ & $0,065 \pm 0,004^{c}$ \\
\hline & & Con harina de avena & $0,235 \pm 0,003^{a}$ & $0,162 \pm 0,001^{b}$ & $0,078 \pm 0,002^{c}$ \\
\hline & & Con harina de caraota & $0,187 \pm 0,005^{a}$ & $0,106 \pm 0,002^{b}$ & $0,056 \pm 0,001^{c}$ \\
\hline & \multirow{3}{*}{ Media } & Sin fibra & $0,291 \pm 0,003^{a}$ & $0,163 \pm 0,004^{b}$ & $0,106 \pm 0,003^{c}$ \\
\hline & & Con harina de avena & $0,292 \pm 0,002^{\mathrm{a}}$ & $0,174 \pm 0,001^{b}$ & $0,130 \pm 0,003^{c}$ \\
\hline & & Con harina de caraota & $0,284 \pm 0,002^{a}$ & $0,134 \pm 0,003^{b}$ & $0,093 \pm 0,006^{c}$ \\
\hline & \multirow{3}{*}{ Distal } & Sin fibra & $0,174 \pm 0,002^{\mathrm{a}}$ & $0,102 \pm 0,003^{b}$ & $0,084 \pm 0,005^{c}$ \\
\hline & & Con harina de avena & $0,224 \pm 0,002^{a}$ & $0,094 \pm 0,002^{b}$ & $0,092 \pm 0,002^{c}$ \\
\hline & & Con harina de caraota & $0,178 \pm 0,003^{a}$ & $0,080 \pm 0,003^{b}$ & $0,099 \pm 0,004^{c}$ \\
\hline
\end{tabular}

$\mathrm{X} \pm \mathrm{DE}$ = promedio más menos desviación estándar. Los valores en una misma fila, con letras distintas, presentan diferencias estadísticamente significativas al aplicar la prueba paramétrica ANOVA de un factor, seguida de la prueba de Tukey $(p<0,05)$.

Tabla 4. Actividad enzimática in vitro de la sacarasa en las tres secciones intestinales, por diferentes fuentes enzimáticas, según tipo de tratamiento

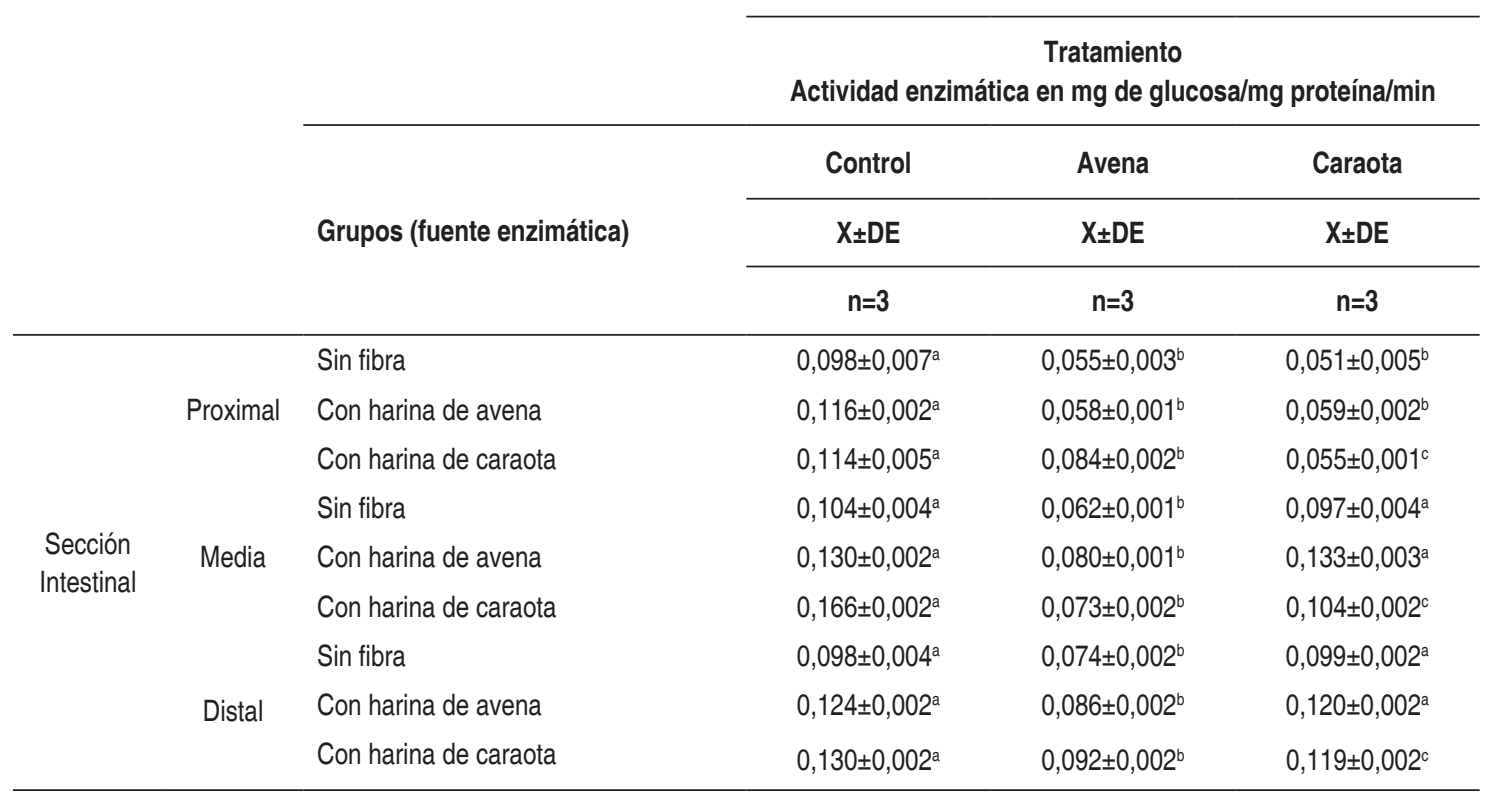

$\mathrm{X} \pm \mathrm{DE}=$ promedio más menos desviación estándar. Los valores en una misma fila, con letras distintas, presentan diferencias estadísticamente significativas al aplicar la prueba paramétrica ANOVA de un factor, seguida de la prueba de Tukey $(p<0,05)$. 
En la sección media, la mayor reducción de la actividad enzimática se observó al añadir residuo de fibra de avena para todas las dietas $(41,1 \%)$, en comparación con la adición de residuo de caraota $(13,9 \%)(p<0,05)$. Este comportamiento en la actividad de la sacarasa también se observó en la sección intestinal distal, donde el descenso de la actividad enzimática en presencia de residuo fibroso de avena alcanzó un 30,6 \% y un $29,2 \%$, respectivamente, en las ratas previamente alimentadas con fibra de avena y caraota.

\section{Actividad in vitro de la lactasa}

En la tabla 5 se muestran los valores de actividad enzimática de la lactasa en presencia de su sustrato natural y cuando al medio de incubación se añadió el residuo fibroso obtenido de la harina de caraota y de la harina de avena, en las tres secciones intestinales estudiadas.

Tabla 5. Actividad enzimática in vitro de la lactasa en las tres secciones intestinales, por diferentes fuentes enzimáticas, según tipo de tratamiento

\begin{tabular}{|c|c|c|c|c|c|}
\hline & & \multirow{4}{*}{ Grupos (fuente enzimática) } & \multicolumn{3}{|c|}{$\begin{array}{l}\text { Tratamiento } \\
\text { Actividad enzimática en } \mathrm{mg} \text { de glucosa/mg proteína/min }\end{array}$} \\
\hline & & & Control & Avena & Caraota \\
\hline & & & $X \pm D E$ & $X \pm D E$ & $X \pm D E$ \\
\hline & & & $n=3$ & $n=3$ & $n=3$ \\
\hline \multirow{9}{*}{$\begin{array}{l}\text { Sección } \\
\text { Intestinal }\end{array}$} & \multirow{3}{*}{ Proximal } & Sin fibra & $0,016 \pm 0,002^{\mathrm{a} 1}$ & $0,005 \pm 0,002^{b}$ & $0,004 \pm 0,002^{b}$ \\
\hline & & Con harina de avena & $0,007 \pm 0,001^{a}$ & $0,005 \pm 0,001^{b}$ & $0,004 \pm 0,001^{c}$ \\
\hline & & Con harina de caraota & $0,029 \pm 0,001^{a}$ & $0,020 \pm 0,002^{b}$ & $0,015 \pm 0,001^{c}$ \\
\hline & \multirow{3}{*}{ Media } & Sin fibra & $0,014 \pm 0,002^{a}$ & $0,007 \pm 0,001^{\mathrm{b}}$ & $0,012 \pm 0,004^{a, b}$ \\
\hline & & Con harina de avena & $0,011 \pm 0,001^{a}$ & $0,007 \pm 0,001^{b}$ & $0,015 \pm 0,002^{c}$ \\
\hline & & Con harina de caraota & $0,053 \pm 0,002^{a}$ & $0,028 \pm 0,002^{b}$ & $0,018 \pm 0,001^{c}$ \\
\hline & \multirow{3}{*}{ Distal } & Sin fibra & $0,009 \pm 0,001^{a}$ & $0,006 \pm 0,002^{a, b}$ & $0,005 \pm 0,002^{a, b}$ \\
\hline & & Con harina de avena & $0,006 \pm 0,001^{a}$ & $0,008 \pm 0,001^{\mathrm{a}}$ & $0,007 \pm 0,002^{\mathrm{a}}$ \\
\hline & & Con harina de caraota & $0,031 \pm 0,002^{\mathrm{a}}$ & $0,017 \pm 0,002^{b}$ & $0,021 \pm 0,002^{b}$ \\
\hline
\end{tabular}

$\mathrm{X} \pm \mathrm{DE}$ = promedio más menos desviación estándar. Los valores en una misma fila, con letras distintas, presentan diferencias estadísticamente significativas al aplicar la prueba paramétrica ANOVA de un factor, seguida de la prueba de Tukey $(p<0,05)$.

En la sección proximal, la adición del residuo fibroso de caraota produjo un descenso significativo de la actividad de la lactasa $(p<0,05)$, que fue mayor en los homogeneizados obtenidos del grupo control de ratas alimentadas previamente con dieta sin fibra $(75,0 \%)$.

En la sección media, la adición del residuo fibroso de avena produjo una disminución significativa $(p<0,05)$ de la actividad de la lactasa en el grupo de ratas previamente alimentadas sin fibra $(50,0 \%)$ y con fibra de avena $(36,4 \%)$. El añadido de fibra de caraota ocasionó una disminución significativa $(p<0,05)$ de $66,0 \%$ en las ratas alimentadas previamente con fibra de caraota, mientras que en el grupo alimentado de forma previa con fibra de avena se observó un aumento significativo $(p<0,05)$ en la actividad enzimática de la lactasa de $36,4 \%$. 
En la sección distal, se pudo observar que la actividad de la lactasa en presencia de residuo de caraota fue la más baja con una reducción promedio del $20 \%$, especialmente en los grupos de ratas alimentadas previamente sin fibra $(44,4 \%)$ y con fibra de caraota $(32,3 \%)$. La adición de avena también ocasionó una reducción de $45,2 \%$ en las ratas alimentadas previamente con fibra de caraota. Finalmente, en las ratas alimentadas con fibra de avena se observó un incremento no significativo tanto con el añadido de residuo fibroso de avena (33,3\%) como con el añadido de fibra de caraota (16,7 \%).

Para cada enzima y sección intestinal se realizó un contraste de medias a través de la prueba de ANOVA de dos vías, en la que se comparó la actividad enzimática entre los grupos experimentales según el tratamiento añadido. En cada una de las nueve comparaciones se pudo identificar que existen diferencias significativas $(p<0,05)$ en la actividad enzimática de acuerdo con el tipo de dieta y el tratamiento realizado, así como con la interacción entre estos dos factores. Por ejemplo, en el caso de la sacarasa medida en la sección media intestinal, se observaron diferencias para el tipo de dieta $(F=367,0 ; p<0,05)$, para el tipo de tratamiento aplicado $(F=1542,0 ; p<0,05)$ y para la interacción entre el tipo de dieta y el tipo de tratamiento $(F=188,02 ; p<0,05)$.

De forma global, se realizó una comparación de las medias para los distintos tipos de enzimas, sección intestinal, dieta previa y tratamiento aplicado, a través de una ANOVA factorial. Se encontraron diferencias significativas para cada factor y sus interacciones. Para el modelo que relacionaba los cuatro factores fijos se obtuvo una $F=25,49$ con una $p<0,05$.

Posteriormente, se aplicó la prueba post-hoc de Tukey para determinar cuáles grupos Ilegaron a ser estadísticamente diferentes $(p<0,05)$. Se pudo constatar que la maltasa tuvo la mayor actividad enzimática $(0,149 \mathrm{mg}$ de glucosa/mg proteína/min), seguida por la sacarasa $(0,096 \mathrm{mg}$ de glucosa/mg proteína/min) y la lactasa $(0,014 \mathrm{mg}$ de glucosa/mg proteína/min). A nivel de la sección intestinal, la mayor actividad enzimática para todas las enzimas se presentó en la sección media $(0,103 \mathrm{mg}$ de glucosa/mg proteína/min; $p<0,05)$, siendo la sección proximal la de menor actividad enzimática $(0,075 \mathrm{mg}$ de glucosa/mg proteína/min).

En todas las enzimas, y en la mayoría de las secciones intestinales, se observó que las actividades enzimáticas de los grupos alimentados previamente con harina de avena $(0,091 \mathrm{mg}$ de glucosa/mg proteína/min) y caraota $(0,088 \mathrm{mg}$ de glucosa/mg proteína/min) llegaron a ser significativamente mayores $(p<0,05)$ a los valores de actividad enzimática general encontrada en el grupo control de ratas alimentadas sin fibra $(0,078 \mathrm{mg}$ de glucosa/mg proteína/min).

En general, el resultado de añadir residuo fibroso de avena y caraota a los homogeneizados de los distintos tipos de dieta, para cada sección intestinal y para cada enzima, fue una inhibición significativa $(p<0,05)$ de la actividad enzimática, en especial con la adición del residuo fibroso de caraota $(0,064 \mathrm{mg}$ de glucosa/mg proteína/ min), seguida por la adición de residuo de avena $(0,071 \mathrm{mg}$ de glucosa/mg proteína/min), en comparación con el grupo control $(0,123 \mathrm{mg}$ de glucosa/mg proteína/min).

Existe una interacción entre los factores de la sección intestinal y el tratamiento aplicado ( $F=597,4$; $p<0,05)$. En la sección proximal, para todos los grupos experimentales, el tratamiento que inhibió en mayor medida la actividad enzimática fue el agregado de residuo fibroso de caraota (maltasa: $67,6 \%$; sacarasa: $49,6 \%$ y lactasa: $55,4 \%$ ), mientras que la inhibición por la adición de fibra 
de avena fue mayor en la porción distal (maltasa: 51,6 \%; sacarasa: 10,7 \% y lactasa: $20 \%$ ). En el segmento medio el efecto fue una disminución proporcional y ajustada a cada enzima, para ambos tratamientos con relación al control.

Al igual que en el modelo anterior, la interacción entre los grupos de tratamientos experimentales $y$ las distintas enzimas fue relevante $(F=5035,0$; $p<0,05)$. La actividad de la maltasa fue inhibida en mayor medida por la adición de residuo de caraota en los tres grupos experimentales, mientras que en la sacarasa el mayor efecto inhibitorio se percibió con el añadido de residuo fibroso de avena. Sin embargo, para la lactasa se observó una disminución dependiente del segmento intestinal, en la que la adición de residuo fibroso de caraota ocasionó una mayor disminución para las secciones proximal $(55,4 \%$ y distal $(20,0 \%)$, mientras que el agregado de avena por su parte inhibió en mayor medida la sección media $(44,5 \%)$.

\section{DISCUSIÓN}

Los valores de fibra dietética total obtenidos para la avena y la caraota cocida son similares a los reportados en la tabla de composición de alimentos venezolana (hojuelas de avena: 10,2 \% y caraotas cocidas: $9,7 \%$ ) (12), y a los valores de la base de datos de composición de alimentos de Estados Unidos (hojuelas de avena: 10,0 \% y caraotas cocidas: $8,7 \%$ ) (13). No obstante, los valores reportados son para fibra dietética total, sin discriminar entre fibra dietética soluble e insoluble. Aunque se ha reportado que la mayoría de la fibra de la caraota es de tipo insoluble, mientras que en la avena prevalece la fibra soluble (14).

A nivel mundial, y en especial en Venezuela, son muy pocos los trabajos realizados in vitro que estudian los posibles efectos de la fibra dietética, 0 de algunos de sus componentes, sobre la activi- dad de las disacaridasas intestinales, utilizando modelos animales. Este campo recibió atención entre las décadas del ochenta y del noventa; pero, actualmente, es poca la literatura encontrada al respecto.

En un estudio sobre el efecto in vitro de las lectinas de Phaseolus vulgaris sobre la actividad de las glucosidasas del borde en cepillo del intestino de ratas, se encontró que tanto la actividad de la maltasa como de la glucoamilasa fueron inhibidas fuertemente por la lectina, en un $80 \%$ aproximadamente, mientras que la actividad de la sacarasa resultó incrementada (15). Estos resultados concuerdan con la presente investigación, en la cual también se observó la disminución en la actividad enzimática de la maltasa en presencia del residuo fibroso de caraota, pero no así para la sacarasa. Otros autores (16) han señalado que el efecto de las lectinas sobre las hidrolasas del borde en cepillo parece ser el resultado de la interacción lectina-receptor en lugar de un efecto intracelular primario. La interacción de las lectinas con la mucosa intestinal se establece a través de la unión específica a receptores presentes en la superficie luminal de los enterocitos.

En ese mismo orden de ideas, un estudio que evaluó la influencia de polisacáridos indigeribles, como pectinas, hemicelulosas y xilanos, sobre la actividad de las enzimas digestivas, encontró que en general la actividad de estas enzimas se veía considerablemente afectada en presencia de los mencionados compuestos fibrosos (6).

Adicionalmente, se ha señalado que el poder inhibitorio varía ampliamente de un residuo a otro. Llegando a concluir que esas diferencias podían ser el reflejo de las distintas proporciones de celulosa, hemicelulosa, pectinas, ligninas y otros materiales indigeribles que constituyen cada residuo fibroso $(17,18)$. Esto podría explicar las diferencias encontradas en el presente estudio 
sobre la actividad de la maltasa cuando se incubó en presencia del residuo fibroso de avena y caraota; esta última ejerció un mayor efecto inhibitorio sobre la actividad de la enzima, lo cual probablemente está relacionado también con las diferentes proporciones de celulosa, hemicelulosa, lignina y otros compuestos fibrosos presentes en la harina de caraota.

Otros estudios han señalado que los componentes de la fracción soluble de la fibra dietética como las gomas, mucílagos, pectinas y $\beta$-glucanos pueden disminuir la adsorción física 0 interferir durante la disponibilidad del sustrato a la enzima (19). Si se toma en consideración que la avena tiene un mayor contenido de fibra soluble que las caraotas, es probable que algunos componentes de esa fracción soluble hayan incidido en la disminución de la actividad de la lactasa en los homogeneizados de los grupos alimentados previamente con avena.

Para tratar de explicar esta reducción de la actividad de la lactasa, una de las propuestas más aceptadas es la captura de la enzima por la matriz fibrosa (20); así como la disminución de la actividad enzimática por un incremento en la viscosidad del medio de reacción mediada por la fibra, lo que se traduce en que al sustrato le toma más tiempo difundir al sitio de la enzima, produciéndose una inhibición de la actividad enzimática (21).

Aunque se desconoce cuál fue la viscosidad del medio cuando se agregaron los residuos fibrosos, sí se ha señalado que la viscosidad parece aumentar en un medio que contiene fibra, especialmente si es fibra dietética soluble. Sin embargo, aceptar cualquiera de estas hipótesis conduce a un mismo resultado: retrasar la difusión efectiva del sustrato hacia el sitio activo de la enzima.
Otros factores como el procesamiento del alimento, las características de la cáscara en el caso de los cereales y las leguminosas, el refinamiento 0 el pulverizado y la cocción o el tipo de cocción, pueden retrasar también la acción de algunas disacaridasas (22).

De igual modo, se ha señalado que otros compuestos como los polifenólicos no se destruyen por el calor, y pueden reducir la utilización de nutrientes. En ese sentido, los taninos, polímeros formados por condensación de monómeros flavan-3 ol y/o flavan 3,4-diol por una sucesiva polimerización, originan productos amorfos como flobafenos y antocianidinas, que pueden formar complejos insolubles con las proteínas reduciendo su utilización digestiva (23); este es uno de los mecanismos propuestos en la disminución 0 inactivación de las enzimas digestivas. Un estudio in vitro similar, en el que se utilizó la variedad tacarigua de Phaseolus vulgaris y el extracto enzimático del intestino completo, mostró como resultado una inhibición de la actividad de la maltasa del 12,5 y del $33 \%$ de inhibición de la sacarasa (24), similar a lo encontrado en el presente estudio. Estos autores proponen a los taninos como posibles inhibidores de las disacaridasas.

Llama la atención que en casi todos los estudios mencionados anteriormente, los ensayos se realizaron con componentes fibrosos purificados (pectinas, hemicelulosa, goma guar, etc), mientras que en la presente investigación se evaluó la actividad de las disacaridasas en presencia del residuo indigerible obtenido de la avena y la caraota por un método enzimático. Esto es importante al momento de hacer extrapolaciones a lo que puede suceder a nivel fisiológico, ya que en una dieta normal se consumen alimentos completos y no componentes aislados de la fibra dietética. 
Evidentemente, el comportamiento particular de la enzima $\beta$-galactosidasa (lactasa) en presencia del residuo fibroso de caraota en la sección intestinal media tuvo un resultado relevante. No se tiene aún una explicación concluyente a este comportamiento; sin embargo, este efecto de estimulación en la actividad de la lactasa podría ser de naturaleza física y estar asociado con un aumento de la accesibilidad del sustrato por el sitio activo de la enzima, que puede resultar de la interacción del residuo fibroso con la matriz proteica correspondiente.

Resultados similares a los descritos en el presente trabajo fueron reportados por Melito (25), quien evaluó en experimentos in vivo e in vitro el efecto de diferentes materiales fibrosos, como celulosas, goma arábiga y afrecho de arroz, sobre la actividad de las disacaridasas de la mucosa intestinal, y observó una reducción significativa de la actividad de maltasa, sacarasa y lactasa. Sin embargo, al incubar las enzimas in vitro en presencia de su sustrato y de los componentes de fibra antes mencionados, observó también que la maltasa y la sacarasa presentaron una inhibición significativa de su actividad, mientras que la lactasa mostró una actividad enzimática sorprendentemente mayor, con relación a las otras disacaridasas.

Un comportamiento similar para la enzima $\beta$-galactosidasa (lactasa) fue encontrado por García (26), quien reportó un aumento sustancial de la actividad de esta enzima cuando fue incubada en presencia de su sustrato natural (lactosa) más la adición de residuo fibroso.

Al respecto, se ha señalado que hay una respuesta adaptativa de la actividad enzimática intestinal a una gran variedad de sustancias, tales como vitaminas, hormonas e incluso a algunas drogas $(27,28)$; por lo que se puede pensar que la fibra dietética en su totalidad, 0 algunos de sus componentes, podrían orientar una adaptación en la actividad enzimática, especialmente en el caso particular del comportamiento observado con la lactasa, cuando se incubó en presencia del residuo fibroso de caraota en la sección intestinal media. Con los presentes resultados no es posible llegar a una conclusión definitiva de este comportamiento enzimático; es evidente la necesidad de diseñar experimentos con tendencias hacia el estudio de la parte molecular de las disacaridasas.

Los hallazgos de este estudio resultan relevantes si se considera que la mayoría de los desórdenes de la digestión y absorción de los disacáridos son el resultado de inadecuados niveles de enzimas digestivas, por lo que la disminución de la actividad enzimática representa un riesgo nutricional y promueve el síndrome de malabsorción.

\section{CONCLUSIONES}

La adición de fibra purificada de avena y caraota produce una disminución significativa de la actividad in vitro de las disacaridasas intestinales, especialmente en presencia del residuo fibroso de caraota. La producción de glucosa de acuerdo con las enzimas estudiadas presenta el siguiente orden: maltasa > sacarasa > lactasa. Así mismo, la mayor actividad enzimática para cada disacaridasa se registra en la sección intestinal media, lo cual evidencia una mayor síntesis de estas enzimas en esta región, en comparación con las secciones proximal y distal.

La ingesta de fibra parece inducir cambios en el epitelio intestinal que son diferentes para cada tipo de fibra y que traen como resultado los cambios observados en la actividad enzimática, por lo cual, se sugiere incluir en los diseños experimentales microscopía óptica o electrónica para verificar la situación de las microvellosidades en cada tratamiento o dieta. 


\section{AGRADECIMIENTOS}

Al Consejo de Desarrollo Científico y Humanístico $(\mathrm{CDCH})$ de la Universidad Central de Venezuela, por el aporte financiero a través del proyecto individual número 09-13-5146-03. Al Laboratorio de Investigaciones Bioquímicas y Nutricionales del Instituto de Biología Experimental (IBE) de la Facultad de Ciencias de la Universidad Central de Venezuela.

\section{- -Referencias}

1. Cummings JH, Engineer A. Denis Burkitt and the origins of the dietary fibre hypothesis. Nutr Res Rev. 2018;31(1):1-15. DOI: $10.1017 /$ S0954422417000117

2. Codex Alimentarius (CODEX). Guidelines on nutrition labeling CAC/GL 2-1985. Joint FAO/WHO Food Standards Programme. Rome: Secretariat of the CODEX Alimentarius Commission. FAO; 2010.

3. The National Academies of Science, Institute of Medicine. Dietary Reference Intakes for energy, carbohydrate, fiber, fat, fatty acids, cholesterol, protein, and amino acids. Washington, DC: National Academies Press; 2002.

4. Ziegler E, Filer L. Conocimientos actuales sobre nutrición. Washington, DC: Organización Panamericana de la Salud; 1997.

5. Amiri M, Naim H. Characterization of mucosal disaccharidases from human intestine. Nutrients. 2017;9(10):pii:E1106. DOI: 10.3390/nu9101106

6. Ferreira-Lazarte A, Olano A, Villamiel M, Moreno F. Assessment of in vitro digestibility of dietary carbohydrates using rat small intestinal extract. J Agric Food Chem. 2017;65(36): 8046-53. DOI: 10.1021/acs.jafc.7b01809

7. Oku T, Tanabe K, Ogawa S, Sadamori N, Nakamura S. Similarity of hydrolyzing activity of human and rat small intestinal disaccharidases. Clin Exp Gastroenterol. 2011;4:155-61. DOI: 10.2147/CEG.S19961

8. Prosky L, Asp N, Schweizer T, DeVries J, Furda I. Determination of insoluble, soluble and total dietary fiber in food products. Chem. 1988;71:1017-23.

9. Lowry O, Rosebrough N, Farr A, Randal R. Methods of determination of proteins. J Biol Chem. 1951;(193):265-66.

10. Trinder P. Methods of enzimatic analysis glucose. Ann Clin Biochem. 1969;6:24-27.

11. National Research Council. Guide for the Care and Use of Laboratory Animals. 8th Edition. Washington, DC: National Academies Press; 2011.

12. Instituto Nacional de Nutrición. Tabla de composición de los alimentos para uso práctico. Caracas: Fondo Editorial Gente de Maíz; 2012.

13. US Department of Agriculture, Agricultural Research Service, Nutrient Data Laboratory. USDA National Nutrient Database for Standard Reference, Release 28; 2018. [Internet]. [Citado febrero de 2018]. Disponible en: https://ndb.nal.usda.gov/ndb/ search/list" https://ndb.nal.usda.gov/ndb/search/list 
14. Dai F, Chau C. Classification and regulatory perspectives of dietary fiber. J Food Drug Anal. 2017;25(1):37-42. DOI: 10.1016/j. jfda.2016.09.006

15. Levy A, Carmona A, Melito C, Santiago J, Contreras L, Tovar J. Efecto antinutricional de lectinas de Phaseolus vulgaris sobre la cascada digestiva de los carbohidratos. Memorias del Instituto de Biología Experimental. 1998;1:41-4.

16. Nciri N, Cho N. New research highlights: Impact of chronic ingestion of white kidney beans (Phaseolus vulgaris $L$. var. Beldia) on small-intestinal disaccharidase activity in Wistar rats. Toxicol Rep. 2017;5:46-55. DOI: 10.1016/j.toxrep.2017.12.016

17. Kohl K, Ciminari M, Chediack J, Leafloor J, Karasov W, McWilliams S, et al. Modulation of digestive enzyme activities in the avian digestive tract in relation to diet composition and quality. J Comp Physiol B. 2017;187(2):339-51. DOI: 10.1007/ s00360-016-1037-6

18. Ahmed F, Urooj A. In vitro hypoglycemic effects and starch digestibility characteristics of wheat based composite functional flour for diabetics. J Food Sci Technol. 2015;52(7): 4530-6. DOI: 10.1007/s13197-014-1470-z

19. Nandini C, Sambaiah K, Salimath P. Effect of dietary fiber on intestinal and renal disaccharidases in diabetic rat. Nutr Research. 2000;20(9):1301-7. DOI: 10.1016/S0271-5317(00)00213-X

20. Bhattarai R, Dhital S, Wu P, Chen X, Gidley M. Digestion of isolated legume cells in a stomach-duodenum model: three mechanisms limit starch and protein hydrolysis. Food Funct. 2017;8(7):2573-82. DOI: 10.1039/c7fo00086c

21. Zhang $Y$, Zhang $H$, Wang $L$, Qian $H$, Qi X, Ding X, et al. The effect of oat $\beta$-glucan on in vitro glucose diffusion and glucose transport in rat small intestine. J Sci Food Agric. 2016;96(2):484-91. DOI: 10.1002/jsfa.7114

22. Maćkowiak K, Torlińska-Walkowiak N, Torlińska B. Dietary fibre as an important constituent of the diet. Postepy Hig Med Dosw (Online). 2016;70:104-9. DOI: 10.5604/17322693.1195842

23. Li M, Hagerman A. Interactions between plasma proteins and naturally occurring polyphenols. Curr Drug Metab. 2013;14(4):432-45. DOI: 10.2174/1389200211314040006

24. Infante RB, García OE, Carmona A, Rivera CJ. Effect of legume dietary fiber on rat disaccharidase activity in vitro. J Nutr Food Sci. 2008;38(4):316-24. DOI: 10.1108/00346650810891379

25. Melito C. Efecto crónico y agudo de la fibra dietética sobre la actividad de las hidrolasas intestinales. Trabajo Especial de Grado. Caracas: Universidad central de Venezuela. Facultad de Ciencias, Escuela de Biología; 1988.

26. García O. Estudio Bioquímico y Nutricional de los materiales indigeribles presentes en cuatro variedades de leguminosas de alto consumo en Venezuela. Trabajo Especial de Grado. Caracas: Universidad central de Venezuela. Facultad de Ciencias; 1994.

27. Neelis E, Olieman J, Hulst J, de Koning B, Wijnen R, Rings E. Promoting intestinal adaptation by nutrition and medication. Best Pract Res Clin Gastroenterol. 2016;30(2):249-61. DOI: 10.1016/j.bpg.2016.03.002

28. Lee B, Rose D, Lin A, Quezada-Calvillo R, Nichols B, Hamaker B. Contribution of the individual small intestinal $\alpha$-glucosidases to digestion of unusual $\alpha$-linked glycemic disaccharides. J Agric Food Chem. 2016;64(33):6487-94. DOI: 10.1021/acs. jafc.6b01816 